\title{
DIFERENCIAS ENTRE LA HEMOGLOBINA OBSERVADA Y ESTIMADA POR HEMATOCRITO Y SU IMPORTANCIA EN EL DIAGNÓSTICO DE ANEMIA EN POBLACIÓN COSTERA VENEZOLANA: ANÁLISIS DEL SEGUNDO ESTUDIO NACIONAL DE CRECIMIENTO Y DESARROLLO HUMANO (SENACREDH)
}

\author{
Jessica Flores-Torres ${ }^{1, a}$, María Echeverría-Ortega ${ }^{1 a}$, Melissa Arria-Bohorquez ${ }^{2, b}$, Glida Hidalgo ${ }^{2, c}$, \\ Carlos Albano-Ramos ${ }^{3, a}$, Rafael Sanz 2 ,a, Alfonso J. Rodríguez-Morales 1,4,5,b
}

\begin{abstract}
RESUMEN
Objetivos. Evaluar las diferencias entre el valor de hemoglobina observada y el valor estimado a partir del hematocrito en el marco del Segundo Estudio Nacional de Crecimiento y Desarrollo Humano de la Población Venezolana (SENACREDH) en el eje centro norte costero del país. Materiales y métodos. Por medio de un muestreo probabilístico multietápico por conglomerados se seleccionó un total de 6004 sujetos que representan 7286781 habitantes del eje Centro Norte Costero (Vargas, Carabobo, Distrito Capital, Aragua y Miranda). Se compararon medias de la hemoglobina observada y hemoglobina estimada (hematocrito/3), usando la prueba t para muestras relacionadas. Se realizaron regresiones lineales entre hemoglobina observada y hematocrito. Resultados. Se observó que el promedio de las diferencias entre la asignadas a la hemoglobina observada y la estimada por el hematocrito fue de $-0,3446 \pm 0,0002(p<0,001)$; sobreestimando significativamente los valores de hemoglobina. Modelos de regresión del hematocrito para la hemoglobina arrojan un valor de $r^{2}=0,87$. Al corregir la estimación, se propone una nueva forma de calcularla, con la siguiente fórmula: hemoglobina estimada $=($ hematocrito/3,135) + 0,257. Conclusiones. Existe una sobreestimación de hemoglobina y, por tanto, subestimación de la prevalencia de anemia a partir del hematocrito; sin embargo, se encuentra una alta correlación entre ambos componentes que permite modelar una mejor estimación de la hemoglobina a partir del hematocrito.
\end{abstract}

Palabras clave: Hemoglobina; Hematocrito; Técnicas de Estimación; Anemia; Venezuela (fuente: DeCS BIREME).

\section{DIFFERENCES BETWEEN OBSERVED AND ESTIMATED BY HEMATOCRIT HEMOGLOBIN AND ITS RELEVANCE IN THE DIAGNOSIS OF ANEMIA AMONG COASTAL POPULATION IN VENEZUELA: ANALYSIS OF THE SECOND NATIONAL STUDY OF HUMAN GROWTH AND DEVELOPMENT (SENACREDH)}

\begin{abstract}
Objectives. To evaluate the differences between the observed hemoglobin levels and those estimated based on hematocrit in the context of the $2^{\text {nd }}$ National Study of Human Growth and Development of the Venezuelan Population (SENACREDH). Materials and methods. 6,004 individuals were chosen by a probabilistic multistage cluster sampling representing 7,286,781 inhabitants from North Central Coastal area (Vargas, Carabobo, Capital District, Aragua and Miranda). Means of observed and estimated hemoglobin (hematocrit/3) were compared, using $t$ test for related samples and linear regression. Results. Mean difference between the values of observed and estimated hemoglobin was $-0.3446 \pm 0.0002(p<0.001)$; significantly overestimating the hemoglobin values. Regression models of hemoglobin on hematocrit showed an $r^{2}=0,87$. In order to correct the estimation, we propose a new formula for calculating hemoglobin based on haematocrit values: estimated hemoglobin=(Haematocrit/3.135) +0.257 . Conclusions: There is an overestimation of hemoglobin levels from hematocrit levels and therefore an underestimation of the prevalence of anemia; however, a high positive correlation between them was found, allowing modeling for achieving a better estimation of the hemoglobin from the hematocrit value.
\end{abstract}

Key words: Haemoglobin; Hematocrit; Estimation techniques; Anemia; Venezuela (source: MeSH NLM).

\footnotetext{
Dirección de Estudios Poblacionales, Centro de Estudios sobre Crecimiento y Desarrollo de la Población (FUNDACREDESA), Ministerio del Poder Popular para las Comunas y Protección Social, Caracas, Venezuela.

2 Dirección de Ciencias Biológicas, Centro de Estudios sobre Crecimiento y Desarrollo de la Población (FUNDACREDESA), Ministerio del Poder Popular para las Comunas y Protección Social, Caracas, Venezuela.

3 Dirección General de Investigación, Centro de Estudios sobre Crecimiento y Desarrollo de la Población (FUNDACREDESA), Ministerio del Poder Popular para las Comunas y Protección Social, Caracas, Venezuela.

4 Cátedra de Salud Pública, Departamento de Medicina Preventiva y Social, Escuela de Medicina Luis Razetti, Facultad de Medicina, Universidad Central de Venezuela, Caracas, Venezuela.

5 Instituto Experimental José Witremundo Torrealba, Núcleo Universitario Rafael Rangel, Universidad de Los Andes, Trujillo, Venezuela.

a Estadístico; ${ }^{\mathrm{b}}$ Médico; ${ }^{\mathrm{c}}$ Nutricionista.
}

Recibido: 04-03-11 Aprobado: 09-03-11 


\section{INTRODUCCIÓN}

La anemia sigue siendo un problema de salud pública a nivel mundial, pero con especial importancia en los países menos desarrollados ${ }^{(1,2)}$. Como una compleja noxa multifactorial, en ella inciden aspectos de diferente índole y naturaleza, entre ellos los más importantes, las enfermedades infecciosas (por ejemplo: parasitosis intestinales, malaria, entre otros) ${ }^{(3,4)}$ y aspectos relacionadas con el déficit de nutrientes ${ }^{(5-7)}$. La anemia se presenta con mayor frecuencia en la población infantil, relacionado con la incidencia y prevalencia de enfermedades que se le asocian a este grupo poblacional ${ }^{(3)}$. En términos geográficos, es mucho más frecuente en zonas rurales y donde existen con mayor importancia enfermedades endémicas y carenciales, así como condiciones de vida más precarias ${ }^{(8,9)}$.

Por estas razones, su diagnóstico y vigilancia epidemiológica activa y pasiva son de gran importancia, especialmente para la formulación de intervenciones sobre sus causas y la prevención del desarrollo de sus efectos ${ }^{(10)}$. En este sentido, el diagnóstico de anemia, aun cuando puede ser muy fácilmente hecho en aquellos lugares que cuentan con laboratorios clínicos básicos, en zonas rurales se basa en muchas ocasiones en los signos y síntomas clínicos y en el uso de estimaciones del valor de hemoglobina a partir del hematocrito (11-15).

A nivel internacional se ha sugerido que los valores de hemoglobina $(\mathrm{Hb})$ pueden ser estimados a partir de la conversión del hematocrito (Htc) / 3 (12-15); sin embargo, varios estudios han indicado una alta imprecisión de dicha estimación ${ }^{(12,13,15)}$, lo cual afectaría el diagnóstico de anemia, conduciendo a sobreestimarla o subestimarla, ello repercute de forma considerable sobre la prevalencia que pueda reportarse.

En Venezuela, solo un estudio previo ha establecido formalmente dicha relación entre los valores de hemoglobina medidos (observados) y los estimados (a partir del hematocrito); en una muestra de 120 pacientes con malaria, procedentes de zonas endémicas, entre 4 a 89 años de edad, evaluados primariamente en un estudio clínico realizado entre los años 2000 y 2002 en Carúpano, en el estado Sucre ${ }^{(12)}$. En ese trabajo se encontró que la diferencia entre la hemoglobina observada y la estimada era significativa $(p<0,05)$, conllevando a una sobrestimación del valor de hemoglobina $y$, por ende, una significativa subestimación de la prevalencia de la anemia ${ }^{(12)}$.

Por estas razones, en el contexto del Segundo Estudio Nacional de Crecimiento y Desarrollo Humano de la Población Venezolana (SENACREDH), con una muestra representativa mayor que los estudios previamente mencionados ${ }^{(12-15)}$, de corte probabilístico, obtenida de 44 municipios de cinco entidades federales del país, se evaluó la relación existente entre la hemoglobina observada y la hemoglobina estimada así como su importancia en el diagnóstico de la anemia.

\section{MATERIALES Y MÉTODOS}

\section{TIPO DE ESTUDIO, ÁREAS DE ESTUDIO Y SELECCIÓN MUESTRAL}

En el marco del Segundo Estudio Nacional de Crecimiento y Desarrollo Humano de la Población Venezolana (SENACREDH), estudio de corte transversal, probabilístico, estratificado, multietápico, que se lleva a cabo entre 2007-2011 por FUNDACREDESA, cuya muestra comprendió 6004 sujetos del eje Centro Norte Costero de Venezuela: Estados Vargas, Carabobo, Aragua, Miranda y Distrito Capital; caracterizadas por ser zonas no endémicas para malaria y con una altitud menor a los 1500 msnm (Figura 1), que representan considerando los factores de expansión - que toma en cuenta, el sexo, la edad y la procedencia - 7286781 habitantes, a los cuales se les realizó evaluación hematológica, incluyendo la medición de la hemoglobina. La estimación de

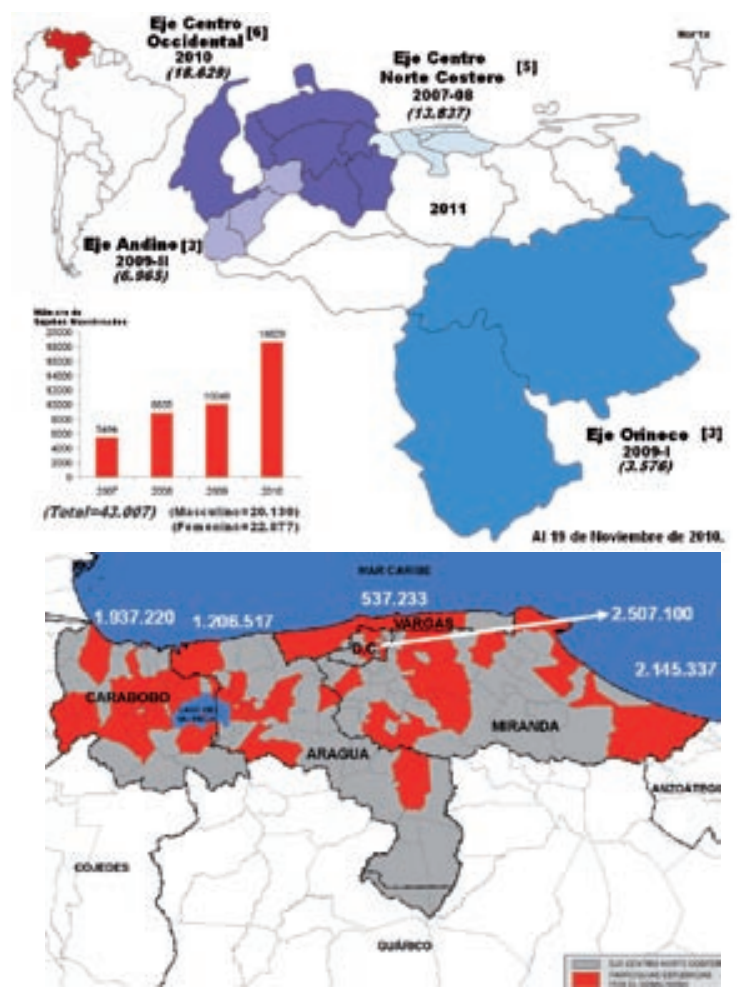

Figura 1. Evolución del levantamiento de la muestra del SENACREDH en Venezuela, incluyen el detalle de las zonas estudiadas del Eje Centro Norte Costero, donde se muestran las estimaciones poblacionales totales para las cuales se analizan los resultados del estudio. 
la potencia estadística posthoc para este estudio fue de $99 \%\left(z_{1-\beta}=17,569\right)$. Los individuos en el estudio fueron aparentemente sanos, población no indígena, nacidos en Venezuela, hijos de padres venezolanos, cuyas edades oscilaban entre 3 meses a menores de 60 años. Las gestantes y los fumadores fueron excluidos de este análisis.

En el SENACREDH se estratificó el país por entidad federal, donde la primera etapa consistió en la selección de conglomerados de segmentos (microárea geográfica que contiene en promedio 200 viviendas de uso familiar). En una segunda etapa se realizó la selección de cinco segmentos dentro de cada conglomerado seleccionado. Esta se realiza con probabilidad proporcional al número de viviendas de uso familiar ocupadas en cada segmento, empleando para ello un método sistemático. La tercera etapa consistió en la selección de cuatro lotes (conglomerados que contienen en promedio 15 viviendas de uso familiar), dentro de cada segmento de la muestra. Se realiza la selección del primer lote de forma aleatoria y los restantes de manera consecutiva. La cuarta etapa consiste en la selección de las personas dentro de las viviendas, de forma sistemática con arranque aleatorio. Esta etapa, se caracteriza por la generación de un marco muestral o listado de todas las personas que habitan las viviendas en cada lote seleccionado con la siguiente información: nombre y apellido, sexo, nacionalidad, edad, ubicación o dirección, número o nombre de la vivienda, parroquia y municipio.

El equipo de personas encargadas de realizar este trabajo se denominan actualizadores, los cuales utilizan como técnica de apoyo, la metodología de actualización cartográfica de áreas, generando así nuevos croquis que indiquen los cambios demográficos, geográficos y urbanísticos presentes. Esta información posteriormente es utilizada para el traslado del resto del equipo de investigación hacia los centros de operaciones para hacer la evaluación, luego se almacena y se depura para así realizar la selección de los sujetos estudio.

\section{MEDICIONES REALIZADAS}

Para la toma de muestra de sangre los sujetos se presentaron en ayunas, la extracción se realizó por punción venosa, en el pliegue del codo, siguiendo el procedimiento descrito en el Manual de técnicas de laboratorio en hematología ${ }^{(16)}$. Una vez tomada la muestra fue llevada al laboratorio y procesada el mismo día.

Para los análisis hematológicos, incluida la medición de la hemoglobina y el hematocrito, se utilizaron tubos al vacío con anticoagulante EDTA Vacuum Diagnostics $₫$. Las determinaciones de hemoglobina y hematocrito se hicieron en los autoanalizadores: Cell-Dyn 1700®,
Cell-Dyn $3200 \AA$, Cell-Dyn $1400 ®$, Celldyn serie Ruby®, Celtac Alfa®, Bayer Advia 60 Hematology Analizer, Coulter ACT $®$, Coulter T660®, Coulter T890®, Coulter STKS ${ }^{\circ}$, ABX Micros OT 60 (Roche Cobas Micros OT60)® y BC-2300 Mindray®.

Los valores de hemoglobina de referencia y valores límites utilizados para niñas, niños, adolescentes y adultos para diagnosticar la anemia, fueron los recomendados por la Organización Mundial de la Salud (OMS) ${ }^{(17) .}$

Todas las mediciones que se realizan en este estudio están sometidas periódicamente a diferentes controles de calidad, con el fin de aumentar la confiabilidad y controlar la variabilidad inter- e intraobservador, añadiendo además, entrenamientos y estandarizaciones con patrones de referencia. En el caso de los estudios de laboratorios hechos, los autoanalizadores empleados se calibran y se les hacen controles de calidad diarios.

\section{ASPECTOS ÉTICOS}

Todos los sujetos firmaron un consentimiento por escrito para participar en el estudio, en el caso de los menores de edad el consentimiento fue otorgado por sus representantes. El presente estudio fue aprobado por un comité de investigación ad hoc del Ministerio del Poder Popular para las Comunas y Protección Social, al cual pertenece el estudio y la institución que lo realiza, FUNDACREDESA.

\section{ANÁLISIS ESTADÍSTICO}

La hemoglobina observada $(\mathrm{g} / \mathrm{dL})$ se comparó con la estimada por el hematocrito (\%), dividido por un factor de tres, tal como lo han reportado estudios previos ${ }^{(12-15)}$. Para establecer la significancia de la diferencia entre los niveles de hemoglobina observada y hemoglobina estimada (hematocrito / 3) (es decir, Hb - Hct / 3) se aplicó una prueba de diferencia de medias para muestras relacionadas (prueba $t$ de Student para muestras relacionadas), habiendo comprobado los supuestos estadísticos de normalidad y homogeneidad previamente.

Aplicando una regresión lineal simple se estableció la relación entre la conversión del hematocrito/3 y la hemoglobina en el diagnóstico de la anemia, posteriormente también se calculó la sensibilidad, especificidad y el coeficiente Kappa ( $\kappa)$ para determinar la concordancia de estos parámetros para el diagnóstico de la anemia y clasificándola por los criterios de Landis y Koch (18).

Los análisis estadísticos se realizaron con el software Statistical Packcage for Social Sciences versión 15 (SPSS, IBM Corporation, Route 100 Somers, NY 10589) y con el paquete "Evaluación de pruebas diagnósticas", 
Tabla 1. Distribución de la hemoglobina observada, la hemoglobina estimada y la diferencia individual en la población en estudio.

\begin{tabular}{cccccc}
\hline $\begin{array}{c}\text { Hb observada } \\
(\mathbf{g} / \mathbf{d L})\end{array}$ & Hb estimada (g/dL) & $\begin{array}{c}\text { Diferencia de } \\
\text { Medias (DM) (g/dL) }\end{array}$ & Error estándar & IC95\% de la DM & $\mathbf{p}^{*}$ \\
\hline 13,5768 & 13,9214 & $-0,3446$ & $<0,0001$ & $-0,3446$ a $-0,3446$ & $<0,001$ \\
\hline
\end{tabular}

IC95\%: intervalo de confianza de 95\%; Hb: hemoglobina. * t para muestras dependientes

v.1.0.2.3 (Unidad de Bioestadística Clínica del Hospital Ramón y Cajal, Madrid, España). Se trabajó con un nivel de significancia de $5 \%$.

\section{RESULTADOS}

Al estimar la hemoglobina a través del hematocrito (Hct / 3), se encontró que la media en la población evaluada era mayor $(13,9214 \mathrm{~g} / \mathrm{dL})$ que la hemoglobina observada $(13,5768 \mathrm{~g} / \mathrm{dL})$. Al comparar dichas medias se observa que el promedio de la diferencia entre las puntuaciones de la hemoglobina observada y la hemoglobina estimada es de $-0,3446( \pm 0,0002059)$ $(p<0,001)$, indicando una significativa sobreestimación de las cifras de hemoblobina a partir de las calculadas a partir del hematocrito (Tabla 1 ).

Al calcular la prevalencia de anemia en la misma población tomando en cuenta los valores de la hemoglobina estimada, se evidencia una significativa subestimación en el diagnóstico de la anemia. Tomando en cuenta la hemoglobina observada para establecer la prevalencia de anemia en la población evaluada se encontró 10,26\% (747 372 sujetos con anemia / 7286781 habitantes) con dicha condición. En cambio, al emplear la hemoglobina estimada la prevalencia de anemia fue solo de $6,06 \%$ (441926 sujetos con anemia / 7286781 habitantes), lo que genera una reducción de $4,20 \%$ en su prevalencia $(p<0,001)$ (Tabla 2). Más aun si lo vemos en términos absolutos; se encuentra una diferencia de 305446 sujetos que no serían diagnosticados con anemia por ese método indirecto (Tabla 2). Dicha diferencia no es uniforme entre los grupos de edad y sexo, pudiendo ser hasta de 9,80\% en menores de 4 años (19,53\% por la observada frente a 9,74 por la estimada) (Figuras 2 y 3 ). Las diferencias en el diagnóstico de anemia entre la hemoglobina observada y estimada son significativas en todos los grupos de edad y en ambos sexos $(p<0,01)$ (Figuras 2 y 3 ).

Por otro lado, al evaluar la concordancia diagnóstica para anemia entre ambas pruebas por medio del estadístico Kappa de Cohen ( $\kappa$ ) se encontró un resultado de 0,620, lo cual indica que la concordancia entre el diagnóstico de anemia realizado por la hemoglobina estimada y el realizado por la hemoglobina observada, es buena, distando mucho de ser absoluta $(\kappa=1,000)$ o cuando menos excelente o muy buena ( $\kappa=0,8100-0,9999)$. Al estimar la sensibilidad de la hemoglobina estimada para el diagnóstico de anemia se encontró que fue de 51,62\% (IC95\% 51,51 a 51,74\%); sin embargo, su especificidad fue alta 99,14\% (IC95\% 99,13 a 99,15\%) (Tabla 2).

Por medio de una regresión lineal se buscó establecer la relación entre el hematocrito y la hemoglobina, de forma tal que se pueda hacer una corrección a la estimación de la hemoglobina a partir del hematocrito; válida para la población de eje Centro Norte Costero de Venezuela, en el caso de que no pueda diagnosticarse la anemia a partir de la medición directa de la hemoglobina. Se obtuvo un $r^{2}=0,872$, lo cual indica que $87,2 \%$ de la variación total de la hemoglobina es explicada por el hematocrito. La prueba de ANOVA ( $F=49.859 .425,32 ; p<0,0001)$ indica que las variables están linealmente y altamente relacionadas, en forma positiva, pero su pendiente no infiere una ecuación donde el valor de la hemoglobina sea igual al hematocrito / 3. Tomando en cuenta dichos resultados se realizó la corrección de la estimación, basándose en la ecuación generada por la regresión lineal simple $(y=\alpha$ $+\beta x)$ que proporciona un buen ajuste, proponiendo una nueva ecuación de la hemoglobina estimada usando el hematocrito, con la siguiente fórmula:

Hemoglobina estimada $=($ Hematocrito $/ 3,135)+0,257$

Tabla 2. Relación entre el diagnóstico de la anemia por la hemoglobina observada y la hemoglobina estimada.

\begin{tabular}{|c|c|c|c|c|c|c|}
\hline \multirow{3}{*}{$\begin{array}{c}\text { Hemoglobina estimada } \\
\text { Anemia } n(\%)\end{array}$} & \multicolumn{6}{|c|}{ Hemoglobina observada } \\
\hline & \multicolumn{2}{|c|}{ Anemia } & \multicolumn{2}{|c|}{ Sin anemia } & \multicolumn{2}{|c|}{ Total $\mathbf{n}(\%)$} \\
\hline & 385821 & $(87,30)$ & 56105 & $(12,70)$ & 441926 & $(6,06)$ \\
\hline Sin anemia $\mathrm{n}(\%)$ & 361551 & $(5,28)$ & 6483304 & $(94,72)$ & 6844855 & $(93,94)$ \\
\hline Total $\mathrm{n}(\%)$ & 747372 & $(10,26)$ & 6539409 & $(89,74)$ & 7286781 & $(100,00)$ \\
\hline
\end{tabular}

$\chi^{2}=3.034 .161,00 ; p<0,001 ; \kappa=0,620 ; p<0,001$.

Para el diagnóstico de la anemia de la hemoglobina estimada al comparársele con el diagnóstico por hemoglobina observada: Sensibilidad=51,6\% $($ IC95\%=51,51 a 51,74); especificidad=99,14\% (IC95\%=99,13 a 99,15); valor predictivo positivo=87,30\% (IC95\%=87,21 a 87,40); valor predictivo negativo $=94,72 \%($ IC95\% $=94,70$ a 94,73$)$ 


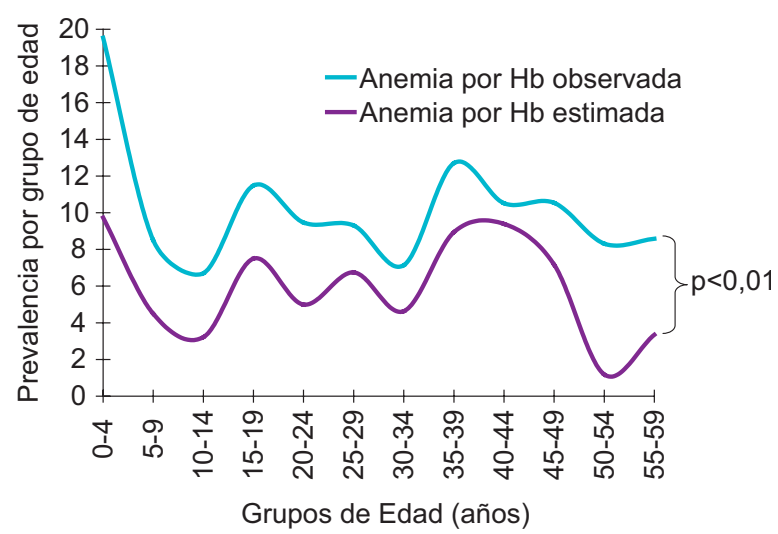

Figura 2. Prevalencia de anemia ajustada por grupo de edad a partir de la hemoglobina observada y hemoglobina estimada del eje norte costero.

\section{DISCUSIÓN}

La relación entre la hemoglobina y el hematocrito ha sido evaluada con interés reciente a fin de tener un método simplificado y económico para el diagnóstico de la anemia, especialmente con fines de pesquisa y estudio de campo de esta y otras condiciones y enfermedades, cuyo principal efecto es el desarrollo de esta, como es el caso de la malaria, entre otras ${ }^{(12-15)}$.

A pesar de que antes del año 2006, en algunos manuales podría haberse sugerido que era posible estimar la hemoglobina usando el hematocrito dividido entre 3 , es en ese año cuando se publica el primer estudio que establece en realidad si hay o no validez en el uso de tal conversión ${ }^{(15)}$. No se encuentra en la literatura internacional -consultando las bases: Index Medicus/ MEDLINE, Scopus, Science Citation Index, SciELO, IMBIOMED- artículos que versen sobre la relación de la hemoglobina y el hematocrito con fines de estimar el valor del primer parámetro a partir del segundo para uso en el diagnóstico de la anemia ${ }^{(1-18)}$.

El mencionado estudio del año 2006, del grupo de Quintó et al. (15), fue una evaluación en una muestra de 5 397 sujetos (2 474 de Mozambique y 2923 de Tanzania) con edades de 2, 5, 8, 12 y 18 meses-en el presente estudio se incluyen todas las edades continuas desde menores de 2 años hasta sujetos menores de 60 años-, con diagnóstico de malaria, donde la hemoglobina fue

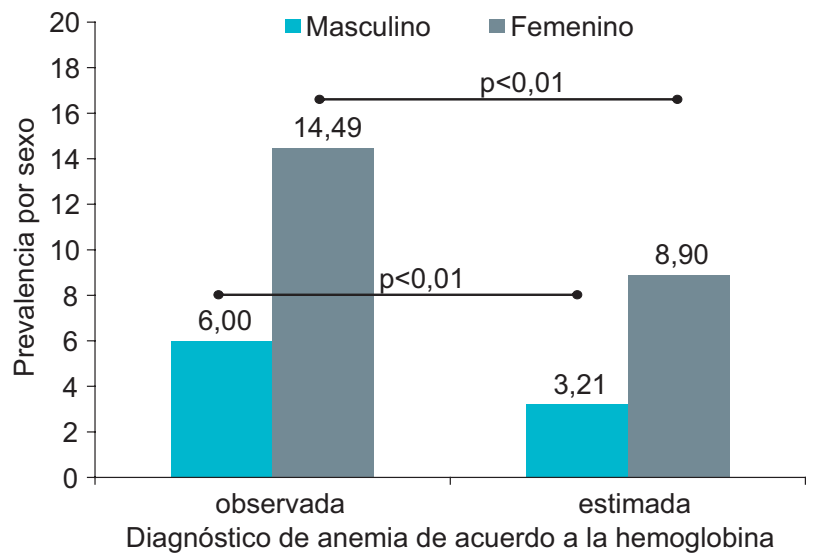

Figura 3. Prevalencia de la anemia por sexo a partir de la hemoglobina observada y hemoglobina estimada del eje norte costero.

determinada con equipos KX-21N y Sysmex F800. Para dicha muestra la concordancia diagnóstica de la anemia entre la hemoglobina observada y la estimada osciló entre $\kappa=0,35$ a 0,60 , lo cual es similar a los resultados presentados por nosotros $(\kappa=0,62)$. En ese mismo trabajo la sensibilidad de la hemoglobina estimada para diagnosticar anemia osciló entre 64 a 92\%, en nuestro trabajo fue incluso menor $(51,62 \%$, IC $95 \% 51,51 \%$ a $51,74 \%$ ). Ese trabajo, como el presente, concluye que los niveles de hemoglobina correspondientes no pueden derivarse con una precisión aceptable utilizando el valor de 3 como factor de conversión único para todas las poblaciones ${ }^{(15)}$.

En el año 2007, un segundo trabajo publicado por el grupo de Carneiro y colaboradores ${ }^{(13)}$, hace una evaluación a una muestra de 1030 sujetos (968 de Ghana y 62 de Tanzania) con edades de 6 a 59 meses de edad (solo menores de 5 años), con sospecha diagnóstica de malaria, donde la hemoglobina fue determinada por haemofotometría (HemoCue ${ }^{\circledR}$ ). Para dicha muestra se observó un sesgo consistente entre la hemoglobina estimada y la observada en $87 \%$ de los casos en los cuales el valor de la estimada era mayor que el de la observada, conllevando a una subestimación de la prevalencia de la anemia que oscilaba entre 10 y $100 \%{ }^{(13)}$. En el presente estudio dicha subestimación alcanzó hasta un $9,8 \%$ de diferencia y la clasificación de casi $60 \%$ de los casos de anemia en forma incorrecta, como libres de dicha condición.

Tabla 3. Regresión lineal entre la hemoglobina observada y la hemoglobina estimada para el diagnóstico de la anemia.

\begin{tabular}{|c|c|c|c|c|c|}
\hline \multirow{2}{*}{ Modelo } & \multicolumn{2}{|c|}{ Coeficientes no estandarizados } & \multirow{2}{*}{$\begin{array}{c}\text { Coeficientes estandarizados } \\
\beta\end{array}$} & \multirow{2}{*}{$\mathbf{t}$} & \multirow{2}{*}{$\mathbf{p}$} \\
\hline & $\beta$ & EE & & & \\
\hline (Constante) & 0,257 & 0,002 & - & 135,534 & $<0,001$ \\
\hline Hb estimada & 0,957 & $<0,001$ & 0,934 & 7061,121 & $<0,001$ \\
\hline
\end{tabular}

EE: error estándar; $\mathrm{Hb}$ : hemoglobina. 
En el año 2007, también se publica el primer trabajo en Venezuela y América Latina el cual en consonancia con el estudio de Quintó ${ }^{(15)}$, como se indicó previamente, fue una evaluación en una muestra de solo 120 sujetos con malaria procedentes de zonas endémicas de 4 a 89 años de edad, evaluados primariamente en un estudio clínico realizado entre los años 2000 y 2002 en Carúpano, Sucre, Venezuela ${ }^{(12)}$. Allí la hemoglobina fue determinada por haemofotometría (HemoCue $\left.{ }^{\circledR}\right)$. Para dicha muestra también se observó que la diferencia entre la medición de la hemoglobina y su estimación por el hematocrito era altamente significativa $(p<0,001)$, indicando que el valor 3 de factor de conversión no era válido y que para esa muestra evaluada se ajustaba mejor con la suma de 0,6215 y división entre 3,1906 para estimar la hemoglobina ${ }^{(12)}$. De manera similar, en el presente trabajo la adición es de 0,257 y la división de 3,135.

Hasta finales del año 2007, tres trabajos ${ }^{(12,13,15)}$, que totalizan 6547 pacientes evaluados indican que es incorrecto o inválido generalizar el uso de la conversión del hematocrito entre 3 para estimar la hemoglobina para todas las poblaciones. Sin embargo, en el año 2008 un estudio publicado por Lee et al. buscó establecer que el uso de la conversión con el factor 3 es válido ${ }^{(14)}$. Dicho estudio fue recopilación de datos procedentes de 85 ensayos clínicos realizados en 25 países (de África y de Asia, en su gran mayoría, solo un país de América Latina, Colombia), con una muestra evaluada de 3254 sujetos con diagnóstico de malaria. Para dicha muestra, usando un modelo de efectos lineales aleatorios, se intentó dar un ajuste para la corrección de la relación entre la hemoglobina y el hematocrito con un $r^{2}$ que osciló entre 0,63 y 0,83, inferior al reportado en el presente trabajo $(0,872)$. Curiosamente el estudio estableció que en promedio, los niveles de hemoglobina estimada eran "ligeramente" mayores que los de la observada cuando se usaba la conversión con el factor 3 , conllevando a una diferencia promedio de $-0,69$ $( \pm 1,3$; IC95\% $-0,75$ a $-0,62)$. En el presente trabajo la diferencia promedio fue menor $(-0,3446)$, pero a diferencia del estudio de Lee et al.(14), en esta evaluación se consideró presentar los valores de prevalencia obtenidos que claramente muestran una subestimación de la anemia ocasionada por la sobrestimación de la hemoglobina a partir de las cifras de hematocrito. En el caso del estudio previamente descrito la diferencia en la prevalencia de anemia debe ser mucho mayor, pero no fue calculada o no fue presentada. Finalmente, ellos sugieren, de todas formas, una nueva ecuación para estimar la hemoglobina usando el hematocrito: hemoglobina estimada = (hematocrito $-5,62) / 2,6^{(14)}$.

Los resultados descritos en el presente trabajo así como los previamente reportados ${ }^{(12 ; 13 ; 15)}$, concluyen que el uso del factor 3 no es preciso, que existen diferencias muy significativas entre la hemoglobina observada y la estimada por dicho método, de forma tal que es necesario evaluar en cada grupo poblacional la validez de nuevos ajustes y correcciones dado que también existen variaciones entre los estudios, especialmente cuando hablamos del contexto poblacional y su aplicación en grandes estudios de pesquisa de anemia. Tanto el trabajo venezolano previo ${ }^{(12)}$, como el presente son los primeros estudios latinoamericanos haciendo la mencionada evaluación que sugiere que debe validarse la relación y establecer el modelo que mejor ajusta a cada población.

Sin embargo, el presente trabajo, que basa sus resultados sobre la inferencia poblacional de 7286781 habitantes, producto de la ponderación de 6004 sujetos, procedentes de 44 municipios de cinco entidades federales de Venezuela, es mucho más representativo y consistente interna y externamente que todos los trabajos previamente publicados hasta el momento ${ }^{(12-15)}$. El ajuste sugerido en el presente trabajo podría permitir su uso en el mencionado ámbito geográfico para el cual es válido estadísticamente.

A pesar de ello, es importante considerar como limitación, que no se pudieron evaluar los niveles de otras variables que pudiesen ayudar en el diagnóstico de la anemia, tales como los niveles de ferritina sérica. Adicionalmente, existen otras limitantes biológicas, que en estudios posteriores esperamos analizar, como el hecho de que en la pubertad hay un incremento importante en la hemoglobina en varones por acción de los andrógenos; sin embargo, la edad de la pubertad es variable por lo cual un varón de 15 años puede ser púber y tener mayor hemoglobina que otro de la misma edad que aún no ha completado la pubertad y tener menor hemoglobina sin ser anémico. Por lo cual podría hacerse una correlación con las variables antropométricas para ajustar según haya ocurrido o no la edad de inicio del estirón de talla.

Otros aspectos como el uso de diferentes definiciones para anemia, como ha sido previamente descrito ${ }^{(19)}$, influyen significativamente en la identificación inapropiada de grupos de riesgo y de los efectos de los programas de intervención a gran escala. Esto también podría ocurrir al sobreestimar los valores de hemoglobina con fórmulas generalizadas a todas las poblaciones.

Finalmente, dado que el SENACREDH es un estudio en curso (Figura 1), el presente es un análisis preliminar, de solo una región del país, que será completado tanto en análisis como en la data en el año 2012. Cuando se hayan levantado y analizado los datos de las restantes 19 entidades federales probablemente se tendrá una base de datos de más de 30000 sujetos que representan a más de 25000000 de habitantes, con lo cual se podrán 
validar los resultados del presente reporte, no solo para cinco entidades federales, sino para el país, incluyendo modelos de regresión ajustando por las distintas edades de los grupos poblacionales (ya mejor representados) y presentando curvas ROC como parte del análisis, entre otras cosas. Todo ello con el fin de mejorar el diagnóstico de la anemia, que representa aún un grave problema de salud pública a nivel mundial, particularmente debido a factores infecciosos y carenciales especialmente en los países menos industrializados ${ }^{(20-22)}$.

\section{AGRADECIMIENTOS}

A la Lic. Carolina Echenagucia, al Diseñador Gráfico José Antonio Ruiz, y en general a la Dirección de Relaciones Institucionales de FUNDACREDESA, por su apoyo en la elaboración gráfica e impresa del trabajo en su presentación en formato póster hecha en la LX Convención Anual de la Asociación Venezolana para el avance de la Ciencias (Aso VAC), 14 al 19 de noviembre de 2010, Cuidad Bolívar, Bolívar, Venezuela, Sesión Parasitología 127-02, Póster CGU-141-129-2010.

\section{Fuentes de Financiamiento}

Ministerio del Poder Popular para las Comunas y Protección Social del Gobierno Bolivariano de Venezuela.

\section{Conflictos de Interés}

Los autores declaran no tener conflictos de interés en la publicación de este artículo.

\section{REFERENCIAS BIBLIOGRÁFICAS}

1. Smith RE, Jr. The clinical and economic burden of anemia. Am J Manag Care. 2010;16(Suppl):S59-66.

2. Tolentino K, Friedman JF. An update on anemia in less developed countries. Am J Trop Med Hyg. 2007;77(1):44-51.

3. Rodriguez-Morales AJ, Sanchez E, Vargas M, Piccolo C, Colina R, Arria M. Anemia and thrombocytopenia in children with Plasmodium vivax malaria. J Trop Pediatr. 2006;52(1):49-51.

4. Rodriguez-Morales AJ, Barbella RA, Case C, Arria M, Ravelo M, Perez H, et al. Intestinal parasitic infections among pregnant women in Venezuela. Infect Dis Obstet Gynecol. 2006;2006:23125.

5. Garcia-Casal MN, Leets I, Bracho C, Hidalgo M, Bastidas G, Gómez A, et al. Prevalence of anemia and deficiencies of iron, folic acid and vitamin B12 in an indigenous community from the Venezuelan Amazon with a high incidence of malaria. Arch Latinoam Nutr. 2008;58(1):12-8.

6. Layrisse M, Garcia-Casal MN, Méndez-Castellano $H$, Jiménez M, Henry O, Chávez JE, et al. Impact of fortification of flours with iron to reduce the prevalence of anemia and iron deficiency among schoolchildren in Caracas, Venezuela: a follow-up. Food Nutr Bull. 2002;23(4):384-9.
7. Hidalgo, G, Hevia P, Macías-Tomei C. Vitamina A, Anemia y Antropometría Nutricional en Preescolares y Escolares Piaroa, Cuenca del Cataniapo, estado Amazonas. Rev Soc Med Quir Hosp Emerg Pérez León. 2009;40(1):6-16.

8. Rodriguez-Morales AJ, Benitez JA, Arria M. Malaria mortality in Venezuela: focus on deaths due to Plasmodium vivax in children. J Trop Pediatr. 2008;54:94-101.

9. Franco-Paredes C, Jones D, Rodriguez-Morales AJ, Santos-Preciado JI. Commentary: improving the health of neglected populations in Latin America. BMC Public Health. 2007;7:11.

10. Illingworth RS. Anaemia and child health surveillance. Arch Dis Child. 1986;61(12):1151-2.

11. Gjorup T, Bugge PM, Hendriksen C, Jensen AM. A critical evaluation of the clinical diagnosis of anemia. Am J Epidemiol. 1986;124(4):657-65.

12. Rodriguez-Morales AJ, Sanchez E, Arria M, Vargas M, Piccolo C, Colina R et al. Hemoglobin and haematocrit: the threefold conversion is also non valid for assessing anaemia in Plasmodium vivax malaria-endemic settings. Malar J. 2007;6:166.

13. Carneiro IA, Drakeley CJ, Owusu-Agyei S, Mmbando B, Chandramohan D. Haemoglobin and haematocrit: is the threefold conversion valid for assessing anaemia in malaria-endemic settings? Malar J. 2007;6:67.

14. Lee SJ, Stepniewska K, Anstey N, Ashley E, Barnes K, Binh TQ, et al. The relationship between the haemoglobin concentration and the haematocrit in Plasmodium falciparum malaria. Malar J. 2008; 7:149.

15. Quinto L, Aponte JJ, Menendez C, Sacarlal J, Aide P, Espasa M, et al. Relationship between haemoglobin and haematocrit in the definition of anaemia. Trop Med Int Health. 2006;11(8):1295-302.

16. Vive J, Aguilar J. Manual de técnicas de laboratorio en hematología. $3^{\text {ra }}$ ed. Madrid: Masson; 2006.

17. World Health Organization (WHO). Worldwide prevalence of anaemia 1993-2005. WHO global database on anaemia. Geneva: WHO; 2008.

18. Landis JR, Koch GG. The measurement of observer agreement for categorical data. Biometrics. 1977;33(1):159-74.

19. Mackerras D, Singh G. The prevalence of anaemia depends on the definition: an example from the Aboriginal Birth Cohort Study. Eur J Clin Nutr. 2007;61(1):135-9.

20. Gulbis B, Eleftheriou A, Angastiniotis M, Ball S, Surrallés $\mathbf{J}$, Castella M, et al. Epidemiology of rare anaemias in Europe. Adv Exp Med Biol. 2010;686(5):375-96.

21. Klemm RD, West KP Jr, Palmer AC, Johnson Q, Randall P, Ranum P, Northrop-Clewes C. Vitamin A fortification of wheat flour: considerations and current recommendations. Food Nutr Bull. 2010;31(1 Suppl):S47-61.

22. Stein J, Hartmann F, Dignass AU. Diagnosis and management of iron deficiency anemia in patients with IBD. Nat Rev Gastroenterol Hepatol. 2010;7(11):599-610.

Correspondencia: Alfonso J. Rodríguez-Morales.

Dirección: Dirección de Estudios Poblacionales, Centro de Estudios sobre Crecimiento y Desarrollo de la Población (FUNDACREDESA), Ministerio del Poder Popular para las Comunas y Protección Social, Caracas, Venezuela.

Correo electrónico: ajrodriguezm_md@hotmail.com 